\title{
HMM-Based Word Alignment in Statistical Translation
}

\author{
Stephan Vogel Hermann Ney Christoph Tillmann \\ Lehrstuhl für Informatik V, RWTH Aachen \\ D-52056 Aachen, Germany \\ \{rogel, ney, tillmann\}@informatik.rwth-aachen.de
}

\begin{abstract}
In this paper, we describe a new model for word alignment in statistical translation and present experimental results. The idea of the model is to make the alignment probabilities dependent on the differences in the alignment positions rather than on the absolute positions. To achieve this goal, the approach uses a first-order Hidden Markov model (HMM) for the word alignment problem as they are used successfully in speech recognition for the time alignment problem. The difference to the time alignment HMM is that there is no monotony constraint for the possible word orderings. We describe the details of the model and test the model on several bilingual corpora.
\end{abstract}

\section{Introduction}

In this paper, we address the problem of word alignments for a bilingual corpus. In the recent years, there have been a number of papers considering this or similar problems: (Brown et al., 1990), (Dagan et al., 1993), (Kay et al., 1993), (Fung et al., 1993).

In our approach, we usc a first-order Hidden Markov model (HMM) (Jelinek, 1976), which is similar, but not identical to those used in speech recognition. The key component of this approach is to make the alignment probabilities dependent not on the absolute position of the word alignment, but on its relative position; i.e. we consider the differences in the index of the word positions rather than the index itself.

The organization of the paper is as follows. After reviewing the statistical approach to machine translation, we first describe the conventional model (mixture model). We then present our first-order HMM approach in full detail. Finally we present some experimental results and compare our model with the conventional model.

\section{Review: Translation Model}

The goal is the translation of a text given in some language $F$ into a target language $E$. For convenience, we choose for the following exposition as language pair French and English, i.e. we are given a French string $f_{1}^{J}=f_{1} \ldots f_{j} \ldots f_{J}^{\prime}$, which is to be translated into an English string $e_{1}^{I}=e_{1} \ldots e_{i} \ldots e_{I}$. Among all possible English strings, we will choose the one with the highest probability which is given by Bayes' decision rule:

$$
\begin{aligned}
\hat{c}_{1}^{I} & =\arg \max _{e_{1}^{I}}\left\{\operatorname{Pr}\left(e_{1}^{I} \mid f_{1}^{J}\right)\right\} \\
& =\arg \max _{e_{1}^{I}}\left\{\operatorname{Pr}\left(e_{1}^{I}\right) \cdot \operatorname{Pr}\left(f_{1}^{J} \mid e_{1}^{I}\right)\right\}
\end{aligned}
$$

$\operatorname{Pr}\left(e_{1}^{I}\right)$ is the language model of the target language, whereas $\operatorname{Pr}\left(f_{1}^{J} \mid e_{1}^{I}\right)$ is the string translation model. The argmax operation denotes the search problem. In this paper, we address the problem of introducing structures into the probabilistic dependencies in order to model the string translation probability $\operatorname{Pr}\left(f_{1}^{J} \mid e_{1}^{I}\right)$.

\section{Alignment Models}

A key issue in modeling the string translation probability $\operatorname{Pr}\left(f_{1}^{J} \mid e_{1}^{I}\right)$ is the question of how we define the correspondence between the words of the English sentence and the words of the French sontence. In typical cases, we can assume a sort of pairwise dependence by considering all word pairs $\left(f_{j}, e_{i}\right)$ for a given sentence pair $\left[f_{1}^{J} ; e_{1}^{I}\right]$. We further constrain this model by assigning each French word to exactly one Iinglish word. Models describing these types of dependencies are referred to as alignment models.

In this section, we describe two models for word alignment in detail:

- a mixture-based alignment model, which was introduced in (Brown et al., 1990);

- an HMM-based alignment model.

In this paper, we address the question of how to define specific models for the alignment probabilities. The notational convention will be as follows. We use the symbol $\operatorname{Pr}($.$) to denote general$ 
probability distributions with (nearly) no specific assumptions. In contrast, for model-based prob-ability distributions, we use the generic symbol $p($.$) .$

\subsection{Alignment with Mixture Distribution}

Ilere, we describe the mixture-based alignment, model in a formulation which is different from the original formulation in (Brown et al., 1990). We will use this model as reference for the IIMMbased alignments to be presented later.

'Ihe model is based on a decomposition of the joint probability for $f_{1}^{J}$ into a product over the probabilitics for cach word $f_{j}$ :

$$
\operatorname{Pr}\left(f_{1}^{J} \mid c_{1}^{I}\right)=p(J \mid I) \cdot \prod_{j=1}^{J} p\left(f_{j} \mid c_{1}^{I}\right),
$$

where, for normalization reasons, the sentence length probability $p(J \mid I)$ has been includel. 'The next step now is to assume a sort of pairwise interaction between the lirench word $f_{j}$ and cach Finglish word $c_{i}, i=1, \ldots l$. These dependencies are captured in the form of a mixture distribution:

$$
\begin{aligned}
p\left(f_{j} \mid e_{1}^{I}\right) & =\sum_{i=1}^{I} p\left(i, f_{j} \mid c_{1}^{I}\right) \\
& =\sum_{i=1}^{I} p(i \mid j, l) \cdot p\left(f_{j} \mid c_{i}\right)
\end{aligned}
$$

Putting everything together, we have the following mixture-based model:

$$
\operatorname{lr} r\left(f_{1}^{J} \mid c_{1}^{l}\right)=p(J \mid I) \cdot \prod_{j=1}^{J} \sum_{i=1}^{I}\left[p(i \mid j, I) \cdot p\left(J_{j} \mid c_{i}\right)\right]
$$

with the following ingredients:

- sentence Jength probability: $p(J \mid I)$;

- mixture alignment probability: $p(i \mid j, l)$;

- translation probability: $p(f \mid c)$.

Assuming a uniform alignment probability

$$
p(i \mid j, I)=\frac{1}{I}
$$

wo arrive at the first model proposed by (Brown ct al., 1990). This model will be referred to as [1BM1 model.

'To train the translation probabilities $p(f \mid c)$, we use a bilingual corpus consisting of sentence pairs $\left[f_{1}^{f_{s}} ; e_{1}^{I_{s}}\right], s=1, \ldots, S$. Using the maximum likelihood criterion, we obtain the following iterative equation (Brown et al., 1990):

$$
\begin{gathered}
\dot{p}(f \mid e)=\frac{A(f, e)}{\sum_{f^{\prime}} A\left(f^{\prime}, e\right)} \quad \text { with } \\
\Lambda(f, e)=\sum_{s}\left(\frac{p(f \mid e)}{\sum_{i=1}^{I_{s}} p\left(f \mid e_{i s}\right)} \sum_{j=1}^{J_{s}} \delta\left(f, f_{j s}\right) \sum_{i=1}^{I_{s}} \delta\left(e, c_{i s}\right)\right)
\end{gathered}
$$

For uniform alignment probabilities, it can be shown (Brown et al., 1990), that there is only one optimum and therefore the WM algorithm (Baum, 1972) always finds the global optimum.

For mixture alignment model with nonuniform alignment probabilities (subsequently referred to as IBM2 model), there are too many alignment parameters $\psi(i \mid j, I)$ to be estimated for small corpora. 'L'herefore, a specific model for the alignment probabilitios is used:

$$
p(i \mid j, l)=\frac{r\left(i-j \frac{l}{J}\right)}{\sum_{i^{\prime}=1}^{l} r\left(i^{\prime}-j \frac{I}{J}\right)}
$$

'This model assumes that the position distance relative to the diagonal line of the $(j, i)$ plane is the dominating factor (see lig. 1). 'Jo train this mode) we use the maximum likelihood criterion in the so-called maximum approximation, i.e. the likelihood criberion covers only the most likely align nnent rather than the set of all alignments:

$$
\operatorname{Pr}\left(f_{1}^{J} \mid e_{1}^{I}\right) \cong \prod_{j=1}^{J} \max _{i}\left[p(i \mid j, l) \cdot p\left(f_{j} \mid e_{i}\right)\right]
$$

In training, this criterion amounts to a sequence of iterations, each of which consists of two steps:

- posilion alignment: Given the model parameters, determine the most likely position alignment.

- parameler estimation: Given the position alignment, i.c. going along the alignment paths for all sentence pairs, perform maximum likelihood estimation of the model parameters; for model-free distributions, these estimates result in relative frequencies.

Due to the nature of the mixture model, there is no interaction between adjacent word positions. Therofore, the optimal position $i$ for each position $j$ can be determined independently of the neighbouring positions. Thus the resulting training procedure is straightforward.

\subsection{Alignment with HMM}

We now propose an IIMM-based alignment model. The motivation is that typically wo have a strong localization effect in aligning the words in parallcl texts (for language pairs from Indoeuropean languages): the words are not distributed arbitrarily over the sentence positions, but tend to form clusters. Fig. 1 illustrates this effect for the language pair German - English.

liach word of the German sentence is assigned to a word of the Finglish sentence. 'The alignments have a strong tendency to preserve the local neightborhood when going from the one language to the other language. In many cases, although not always, there is an even stronger restriction: the difference in the position index is smaller than 3. 


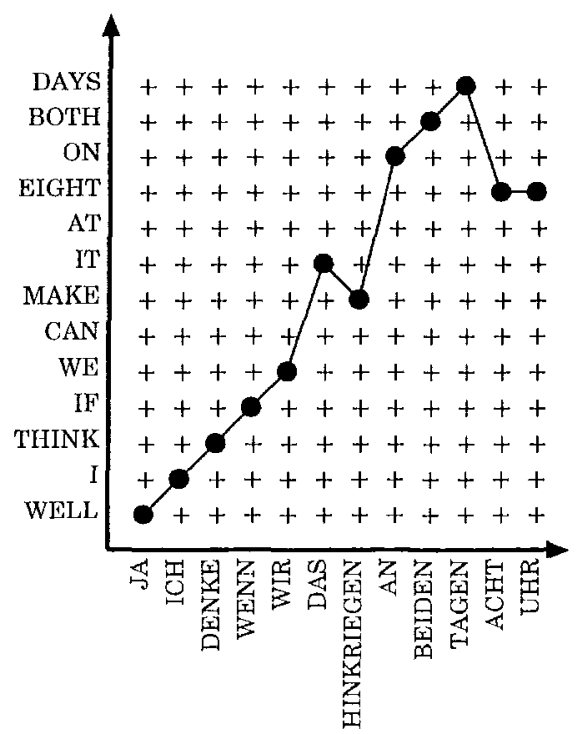

Figure 1: Word alignment for a German - English sentence pair.

To describe these word-by-word alignments, we introduce the mapping $j \rightarrow a_{j}$, which assigns a word $f_{j}$ in position $j$ to a word $e_{i}$ in position $i=a_{j}$. The concept of these alignments is similar to the ones introduced by (Brown ef al., 1990), but we will use another type of dependence in the probability distributions. Looking at such alignments produced by a human expert, it is evident that the mathematical model should try to capture the strong dependence of $a_{j}$ on the previous alignment. Therefore the probability of alignment $a_{j}$ for position $j$ should have a dependence on the previous alignment $a_{j-1}$ :

$$
p\left(a_{j} \mid a_{j-1}, I\right),
$$

where we have included the conditioning on the total length $I$ of the English sentence for normalization reasons. $\Lambda$ similar approach has been chosen by (Dagan et al., 1993). Thus the problem formulation is similar to that of the time alignment problem in speech recognition, where the so-called IIidden Markov models have been successfully used for a long time (Jelinek, 1976). Using the same basic principles, we can rowrite the probability by introducing the 'hidden' alignments $a_{1}^{J}:=a_{1} \ldots a_{j} \ldots a_{J}$ for a sentence pair $\left[f_{1}^{J} ; e_{1}^{I}\right]$ :

$$
\begin{aligned}
\operatorname{Pr}\left(f_{1}^{J} \mid e_{1}^{I}\right) & =\sum_{a_{1}^{J}} \operatorname{Pr}\left(f_{1}^{J}, a_{1}^{J} \mid e_{1}^{I}\right) \\
& =\sum_{a_{1}^{J}} \prod_{j=1}^{J} \operatorname{Pr}\left(f_{j}, a_{j} \mid f_{1}^{j-1}, a_{1}^{j-1}, e_{1}^{I}\right)
\end{aligned}
$$

So far there has been no basic restriction of the approach. We now assume a first-order dependence on the alignments $a_{j}$ only:

$$
\operatorname{Pr}\left(f_{j}, a_{j} \mid f_{1}^{j-1}, a_{1}^{j-1}, e_{1}^{I}\right)
$$

$$
\begin{aligned}
& =p\left(f_{j}, a_{j} \mid a_{j-1}, e_{1}^{I}\right) \\
& =p\left(a_{j} \mid a_{j-1}, I\right) \cdot p\left(f_{j} \mid e_{a_{j}}\right)
\end{aligned}
$$

where, in addition, we have assumed that the translation probability depends only on $a_{j}$ and not on $a_{j-1}$. Putting everything together, we have the following IIMM-based model:

$\operatorname{Pr}\left(f_{1}^{J} \mid e_{1}^{I}\right)=\sum_{a_{1}^{J}} \prod_{j=1}^{J}\left[p\left(a_{j} \mid a_{j-1}, l\right) \cdot p\left(f_{j} \mid e_{a_{j}}\right)\right]$

with the following ingredients:

- HMM alignment probability: $p\left(i \mid i^{\prime}, l\right)$ or $p\left(a_{j} \mid a_{j-1}, I\right)$;

- translation probability: $p(f \mid e)$.

In addition, we assume that the HMM align ment probabilities $p\left(i \mid i^{\prime}, I\right)$ depend only on the jump width $\left(i-i^{\prime}\right)$. Using a set of non-negative parameters $\left\{s\left(i-i^{\prime}\right)\right\}$, we can write the HMM alignment probabilities in the form:

$$
p\left(i \mid i^{\prime}, I\right)=\frac{s\left(i-i^{\prime}\right)}{\sum_{l=1}^{l} s\left(l-i^{\prime}\right)}
$$

This form ensures that for each word position $i^{\prime}, i^{\prime}=1, \ldots, I$, the HMM alignment probabilitics satisfy the normalization constraint.

Note the similarity between Equations (2) and (5). The mixturc model can be interpreted as a zeroth-order model in contrast to the first-order HMM model.

As with the IBM2 model, we use again the maximum approximation:

$\operatorname{Pr}\left(f_{1}^{J} \mid e_{1}^{I}\right) \cong \max _{a_{1}^{J}} \prod_{j=1}^{J}\left[p\left(a_{j} \mid a_{j-1}, I\right) \cdot p\left(f_{j} \mid e_{a_{j}}\right)\right]$

In this case, the task of finding the optimal alignment is more involved than in the case of the mixture model (IBM2). Therefore, we have to resort to dynamic programming for which we have the following typical recursion formula:

$Q(i, j)=p\left(f_{j} \mid e_{i}\right) \max _{i^{\prime}=1, \ldots, I}\left[p\left(i \mid i^{\prime}, I\right) \cdot Q\left(i^{\prime}, j-1\right)\right]$

Here, $Q(i, j)$ is a sort of partial probability as in time alignment for specch rocognition (Jelinek, 1976).

\section{Experimental Results}

\subsection{The Task and the Corpus}

'The models were tested on several tasks:

- the Avalanche Bulletins published by the Swiss Federal Institute for Snow and Avalanche Rescarch (SFISAR) in Davos, Switzerland and made available by the European Corpus Initiative (FCI/MCI, 1994);

- the Verbmobil Corpus consisting of spontaneously spoken dialogs in the domain of appointment scheduling (Wahlster, 1993); 
- the Julrans Corpus which contains typical phrases from the tourists and travel domain. (LuTrans, 1996).

Table 1 gives the details on the size of the corpora and their vocabulary. It should be noted that in all these three cases the ratio of vocabu lasy size and number of ruming words is not very favorable.

Table 1: Corpora

\begin{tabular}{|c|c|c|c|}
\hline Gorpus & Tallguage & Words & Voc. Size \\
\hline \multirow[t]{2}{*}{ Avalanche } & lrench & 62849 & 1993 \\
\hline & German & 44805 & 2265 \\
\hline \multirow[t]{2}{*}{ EuTrans } & Sjatnish & 13768 & 2008 \\
\hline & Hinglish & 15888 & 1630 \\
\hline \multirow[t]{2}{*}{ Verbmobil } & Gorman & 150279 & 4017 \\
\hline & Funglish & 154727 & 2413 \\
\hline
\end{tabular}

lior several yoars between 83 and 92 , the $\Lambda$ valanche Bulletins are avalable for both Gernuan and lirench. 'The following is a typical sentlence pair from the corpus:

Bei zuerst rechl holen, spater tieferen 'lemperaturen sind von Samstag bis 1)icnstag morgen auf der $\Lambda l_{\text {pennordseite }}$ und an $\Lambda$ pen. hauptkanm oberhalb $2000 \mathrm{~m} 60$ bis $80 \mathrm{~cm}$ Neuschnoe gefallen.

Par des tempóratures d' abord élevés, puis plus basses, 60 à $80 \mathrm{~cm}$ de neige sont tombs de simedi a mardi mabiu sur le versant nord et la crôte des $\Lambda$ pes auldessus de $2000 \mathrm{~m}$.

An example from the Verbmobil corpus is given in ligure 1.

\subsection{Training and Rosults}

liach of the three corpora were ased to train both alignment models, the mixt,ure-based alignment model in Ha.(1) and the HMM-based alignment model in Ha.(1). Here, we will consider the experimental tess on the Avalanche corpus in more detail. 'The training procedure consisted of the following steps:

- Initialization training: IHMMI noclel trained for 10 iterations of the WM algorithm.

- Refinement tratning: 'The transtation probatbilities from the initialization training were used to initialize both the II3M2 model and the II M M-based alignment model

IBM2 Model: 5 iterations using the maximum approximation (b.a.(3))

HMM Model: 5 iterations using the max.

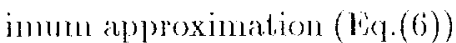

'l'he resulting per] lexity (inverso greometric average of the likelihoods) for the different models are given in the 'Jables 2 and 3 for the $A$ valanche corpus. In addition to the total perplexity, which is the global optimization criterion, the tables also show the perplexities of the translation probabilities and of the alignment probabilities. The last line in Table 2 gives the perplexity measures when applying the maximum approximation and computing the perplexity in this approximation. 'These values are equal to the ones after initializing the II3M2 and IIMM nodels, as they should be. From Table 3, we can soe that the mixture alignment gives slightly better perplexity values for the transtation probabilities, whoreas the $11 M M$ nodel produces a smaller perplexity for the alignment probabilitics. In the calculation of the perplexities, the sentence length probability was not included.

'Jable 2: IBM I: 'Translation, alignment and total perplexity as a function of the iteration.

\begin{tabular}{|r|r|r|r|}
\hline Tteration & Translation & Alignment & Total \\
\hline 0 & 99.30 & 20.07 & 1994.00 \\
1 & 3.72 & 20.07 & 74.57 \\
2 & 2.67 & 20.07 & 53.62 \\
$\ldots$ & $\ldots$ & $\ldots$ & $\ldots$ \\
9 & 1.87 & 20.07 & 37.55 \\
10 & 1.86 & 20.07 & 37.36 \\
\hline Max. & 3.88 & 20.07 & 77.95 \\
\hline
\end{tabular}

'able 3: 'Iranslation, alignment and total perplexity as a function of the iteration for the IBM2 $(\Lambda)$ and tho II MM model (B)

\begin{tabular}{|c|c|r|r|r|}
\hline$\Lambda$ & Iter. & Trumslation & Nligninent & Total \\
\hline & 0 & 3.88 & 20.07 & 77.95 \\
& 1 & 3.17 & 10.82 & 34.27 \\
& 2 & 3.25 & 10.15 & 33.03 \\
& 3 & 3.22 & 10.10 & 32.48 \\
& 1 & 3.20 & 10.06 & 32.18 \\
& 5 & 3.18 & 10.05 & 32.00 \\
\hline 13 & 0 & 3.88 & 20.07 & 77.95 \\
& 0 & 3.37 & 7.99 & 26.98 \\
& 1 & 3.46 & 6.17 & 21.36 \\
& 2 & 3.47 & 5.90 & 20.48 \\
& 3 & 3.46 & 5.85 & 20.24 \\
& 4 & 3.45 & 5.81 & 20.18 \\
\hline
\end{tabular}

Another interesting question is whether tho IIMM aligntment model helps in finding good and sharply focussed word-to-word correspondences. As an example, lable 4 gives a comparison of the translation probabilities $p(f / e)$ between tho mixture and the IIMM alignment model for the Creman word Alpensildhang. 'I'he counts of the words are given in brackets. There is virtually no difference between the translation tables for the two models (IBM2 and IIMM). But in gencral, the IIMM model seems to give slightly better results in the cases of German compound words like Alpensüdhang - versant sud des Alpes which require function words in the translation. 
Table 4: Alpensüdhang.

\begin{tabular}{|l|l|l|}
\hline IBM1 & Alpes (684) & 0.171 \\
& des (1968) & 0.035 \\
& le (1419) & 0.039 \\
& sud (416) & 0.427 \\
& sur (769) & 0.040 \\
& versant (431) & 0.284 \\
\hline IBM2 & Alpes (684) & 0.276 \\
& sud (416) & 0.371 \\
& versant (431) & 0.356 \\
\hline HMM & Alpes (684) & 0.284 \\
& des (1968) & 0.028 \\
& sud (416) & 0.354 \\
& versant (431) & 0.333 \\
\hline
\end{tabular}

This is a result of the smoother position alignments produced by the HMM model. A pronounced example is given in Figure 2. 'The problem of the absolute position alignment can be demonstrated at the positions (a) and (c): both Schneebrettgefahr und Schneeverfrachtungen have a high probability on neige. 'The IBM2 models chooses the position near the diagonal, as this is the one with the higher probability. Again, Schneebrettgefahr generates de which explains the wrong alignment near the diagonal in (c).

However, this strength of the HMM model can also be a weakness as in the case of est developpe - ist ... entstanden (see (b) in Figure 2. The required two large jumps are correctly found by the mixture model, but not by the HMM model. These cases suggest an extention to the HMM model. In general, there are only a small number of big jumps in the position alignments in a given sentence pair. Therefore a model could be useful that distinguishes between local and big jumps.

The models have also been tested on the Verbmobil Translation Corpus as well as on a small Corpus used in the EuTrans project. The sentences in the EuTrans corpus are in general short phrases with simple grammatical structures. However, the training corpus is very small and the produced alignments are generally of poor quality. There is no marked difference for the two align ment models.

Table 5: Perplexity results for (a) Eu'Trans and (b) Verbmobil Corpus.

\begin{tabular}{|c|r|r|r|r|r|}
\hline & Model & Iter. & Transl. & Align. & Total \\
\hline a & IBM1 & 10 & 2.610 & 6.233 & 16.267 \\
& IBM2 & 5 & 2.443 & 4.003 & 9.781 \\
& HMM & 5 & 2.461 & 3.934 & 9.686 \\
\hline b & IBM1 & 10 & 4.373 & 10.674 & 46.672 \\
& IBM2 & 5 & 4.696 & 6.538 & 30.706 \\
& HMM & 5 & 4.859 & 5.452 & 26.495 \\
\hline
\end{tabular}

The Verbmobil Corpus consists of spontaneously spoken dialogs in the domain of appointment scheduling. The assumption that every word in the source language is aligned to a word in the target language breaks down for many sentence pairs, resulting in poor alignment. This in turn affects the quality of the translation probabilities.

Several extensions to the current IIMM based model could be used to tackle these problems:

- The results presented here did not use the concept of the empty word. For the HMMbased model this, however, requires a secondorder rather than a first-order model.

- We could allow for multi-word phrases in both languages.

- In addition to the absolute or relative alignment positions, the alignment probabilities can be assumed to depend on part of speech tags or on the words themselves. (confer model 4 in (Brown et al., 1990)).

\section{Conclusion}

In this paper, we have presented an HMM-based approach for modelling word alignments in parallel texts. The characteristic feature of this approach is to make the alignment probabilities explicitly dependent on the alignment position of the previous word. Wo have tested the model successfully on real data. The HMM-based approach produces translation probabilities comparable to the mixture alignment model. When looking at the position alignments those generated by the HMM model are in general much smoother. This could be especially helpful for languages such as German, where compound words are matched to several words in the source language. On the other hand, large jumps due to different word orderings in the two languages are successfully modeled. We are presently studying and testing a multilevel HMM model that allows only a small number of large jumps. The ultimate test of the different alignment and translation models can only be carried out in the framework of a fully operational translation system.

\section{Acknowledgement}

This research was partly supported by the German Federal Ministery of Education, Science, Research and Technology under the Contract Number 01 IV $601 \mathrm{~A}$ (Verbmobil) and under the Esprit Research Project 20268 (Eu'lrans).

\section{References}

L. F. Baum. 1972. An inequality and associated maximization technique in statistical estimation of probabilistic functions of a Markov process. Inequalities, $3: 1-8$. 


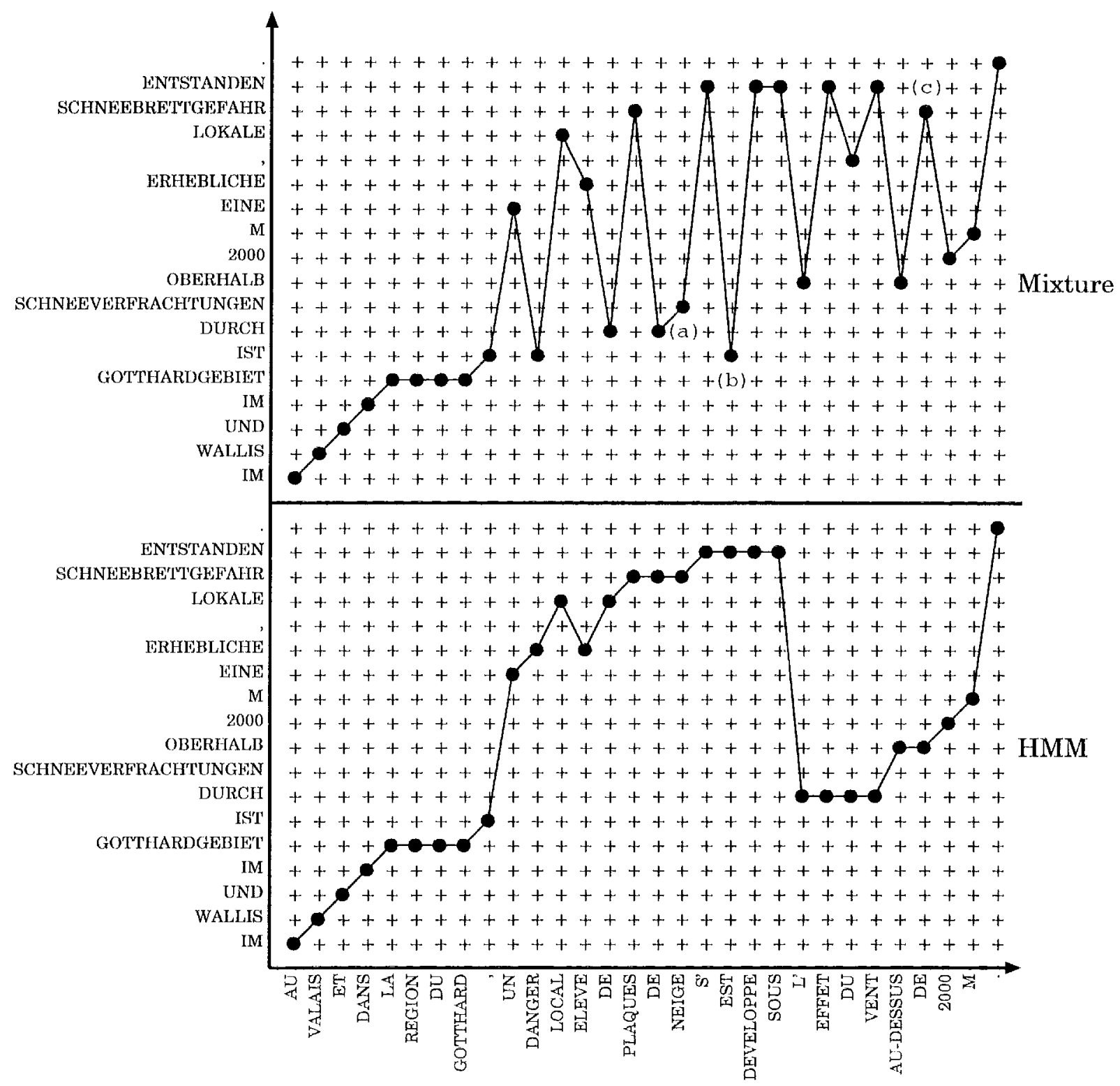

Figure 2: Alignments generated by the IBM 2 and the IIMM model.

Peter $F^{2}$, Brown, Vincent J. Della Pietra, Stcphen A. Dolla Pietra, and Robert 1. Mercer. 1993. The Mathematics of Statistical Machine 'Translation: Parauneter Listimation. Computational Linguistics, 19(2):263--311.

Jolo Dagan, Ken Church, and William $\Lambda$. Gale. 1993. Robust Bilingual Word Alignment for: Machine Aicled 'Translation Proceedings of the Workshop on Very Large Corpora, Columbus, Ohio, $1-8$

ECI/MCI: The Furopean Corpus Initiative Multilingual Corpus 1. 1994. Association for Computational linguistics.

lithans. 'The Definition of a M'I' 'Task. 'Technical Report, lin'lrans Project. 1996 (lorthcomming). Depto. de Sistemas Informaticos y
Computacion (DSIC), Universidad Politecnica de Valencia.

Pascale I'ung, and Kenneth Ward Church. 1994. $K$ vec: $\Lambda$ new approach for aligning parallel texts. Procedings of COLING 9/, 1096-1102, Kyoto, Japan.

Froderik Jelinek. 1976. Speech Recognition by Statistical Methods. Irocedings of the lell', Vol. 64, 532-556, April 1976.

Martin Kay, and Martin Röscheisen. 1993. 'TextTranslation Alignment. Compulational linguistics, 19(1):121-142

Wolfgang Wahlster. 1993. Verbmobil: 'Translation of lace-to-Face Dialogs. Procedings of the MT Summit IV, 127-135, Kobe, Japan. 